\title{
Notas sobre a ocorrência de Tityus serrulatus Lutz \& Mello, 1922 (Scorpiones, Buthidae) no oeste do Rio Grande do Sul, Brasil
}

\author{
Luis Roberval Bortoluzzi ${ }^{1,3}$, Marcus Vinícius Morini Querol ${ }^{2}$ \& Enrique Querol ${ }^{I}$
}

Biota Neotropica v7 (n3) - http://www.biotaneotropica.org.br/v7n3/pt/abstract?article +bn00707032007

\author{
Recebido em 16/01/07 \\ Versão reformulada recebida em 05/07/07 \\ Publicado em 01/09/07
}

${ }^{1}$ Museu de Ciências Naturais, Curso de Ciências Biológicas, Faculdade de Filosofia, Ciências e Letras, Pontifícia Universidade Católica do Rio Grande do Sul-PUCRS, Campus Uruguaiana, Rodovia BR 472, Km 7, CEP 97500-970, Uruguaiana, RS, Brasil

${ }^{2}$ Universidade Federal Do Pampa - UNIPAMPA ITAQUI,

Rua Euclides Aranha, $n^{\circ}$ 1288, Bairro Centro, CEP 97650-000, Itaqui, RS, Brasil

${ }^{3}$ Autor para correspondência: Luis Roberval Bortoluzzi, e-mail: lbortoluzzi@gmail.com

\begin{abstract}
Bortoluzzi, L.R., Querol, M.V.M. \& Querol, E. Notes on the occurrence of Tityus serrulatus Lutz \& Mello, 1922 (Scorpiones, Buthidae) in the Western areas of Rio Grande do Sul, Brazil. Biota Neotrop. Sep/Dez 2007 vol. 7, no. 3 http://www.biotaneotropica.org.br/v7n3/pt/abstract?article+bn00707032007. ISSN 1676-0603.

The yellow scorpion Tityus serrulatus is recorded for the first time for the western Rio Grande do Sul State, Brazil. Two specimens were collected in the municipality of Uruguaiana ( $29^{\circ} 45^{\prime} 23^{\prime \prime} \mathrm{S}$ and $57^{\circ} 05^{\prime} 37^{\prime}$ ' W) in june 2006. The occurrence of specimens of T. serrulatus in Rio Grande do Sul represents an increase of $2127 \mathrm{~km}$ in relation to his known distribution area.

Keywords: Tityus serrulatus, yellow scorpion, distribution, Rio Grande do Sul.

Resumo

Bortoluzzi, L.R., Querol, M.V.M. \& Querol, E. Notas sobre a ocorrência de Tityus serrulatus Lutz \& Mello, 1922 (Scorpiones, Buthidae) no oeste do Rio Grande do Sul, Brasil. Biota Neotrop. Sep/Dez 2007 vol. 7, no. 3 http://www.biotaneotropica.org.br/v7n3/pt/abstract?article+bn00707032007. ISSN 1676-0603.

O escorpião amarelo Tityus serrulatus é registrado pela primeira vez para oeste do Rio Grande do Sul. Foram coletados dois espécimes no município de Uruguaiana $\left(29^{\circ} 45^{\prime} 23^{\prime \prime} \mathrm{S}\right.$ e $57^{\circ} 05^{\prime} 37^{\prime}$ ' W) em junho de 2006. A ocorrência dos espécimes de T. serrulatus no Rio Grande Sul representa um aumento de $2.127 \mathrm{~km}$ em sua área de distribuição conhecida.
\end{abstract}

Palavras-chave: Tityus serrulatus, escorpião amarelo, distribuição, Rio Grande do Sul. 


\section{Introdução}

Os escorpiões existem comprovadamente há mais de 400 milhões de anos (fósseis do Carbonífero), sendo, portanto, considerados os aracnídeos mais antigos que se conhece (Cruz, 1994). Embora represente um grupo bastante homogêneo quanto às suas características morfológicas, a ordem Scorpiones pode ser considerada razoavelmente diversa, apresentando 1500 espécies conhecidas, distribuídas em 14 famílias e 163 gêneros (Fet et al. 1998, Soleglad \& Fet 2003).

Conforme Soleglad \& Fet (2003) a ordem Scorpiones apresenta ampla distribuição geográfica, estando representada em todos os continentes, com exceção da Antártida. No Brasil, a escorpiofauna é representada por cinco famílias, Liochelidae, Euscorpiidae, Chactidae, Bothriuridae e Buthidae, abrangendo 17 gêneros e 86 espécies consideradas atualmente válidas (Lourenço \& Eickstedt 2003).

O gênero Tityus Koch, 1836, inclui o maior número de espécies descritas da ordem Scorpiones (Fet et al. 2000); apresenta uma ampla distribuição geográfica na América do Sul e, também no Brasil, é o gênero mais especioso, estando representado por 35 espécies (Lourenço 2002).

No Rio Grande do Sul a escorpiofauna é representada por um reduzido número de espécies.

Do gênero Tityus estão registradas três espécies, o escorpião marrom T. bahiensis (Funasa 2001, Cupo et al. 1994a); o escorpião manchado T. costatus, (Lourenço \& Eickstedt 1988) e T. serrulatus com um registro para a região metropolitana de Porto Alegre.

O escorpião amarelo, T. serrulatus é considerado a espécie mais perigosa da América do Sul (Bucaretchi et al. 1994; Cupo et al. 1994b; Eickstedt et al. 1994; Freire-Maia et al. 1994). Esta espécie é endêmica do Brasil com uma área de distribuição conhecida para os estados da Bahia, Espírito Santo, Minas Gerais, Rio de Janeiro, São Paulo, Paraná, Distrito Federal e Goiás, (Fundacentro 2001).

Este trabalho tem por objetivo registrar a ocorrência de T. serrulatus para a região oeste do Rio Grande do Sul.

\section{Material e Métodos}

Dois exemplares de T. serrulatus (Figura 1) foram coletados em junho de 2006, no município Uruguaiana localizado a $29^{\circ} 45^{\prime} 23^{\prime}$ 'S e $57^{\circ} 05^{\prime} 37^{\prime}$ 'W, no oeste do Estado do Rio Grande do Sul (Figura 2), área de fronteira entre Brasil, Argentina e Uruguai.

Este município é caracterizado por apresentar uma vegetação do tipo estepe (planalto da campanha), ocorrendo áreas com solos pedrosos; relevo de morfologia plana, sub-horizontalizada; altitude média de $74 \mathrm{~m}$ acima do nível do mar, (Brasil 1986). A região apresenta um clima temperado úmido, com temperaturas médias mensais que variam de 23 a $27^{\circ} \mathrm{C}$, no período de calor, e de 14 a $15^{\circ} \mathrm{C}$, no período frio (Brasil 1973).

Os espécimes foram capturados nas instalações de uma empresa transportadora, localizada próximo à periferia da cidade.

Os animais foram fixados com formol a $10 \%$ e conservados em álcool $70 \%$, medidos com um paquímetro e observados em lupa, para a identificação taxonômica. Os exemplares foram tombados na coleção científica do Museu de Ciências Naturais da Pontifícia Universidade Católica do Rio Grande do Sul - Campus Uruguaiana sob número de registro: MCN-PUC 77-22-14.

\section{Resultados e Discussão}

Um dos espécimes mediu $68,0 \mathrm{~mm}$ e o outro 72,0 $\mathrm{mm}$, ambos apresentam colorido amarelo-claro nas pernas, pedipalpos (com dedo fino e granulações no dedo móvel) e cauda. O tronco, dedos e parte final do último segmento da cauda são escuros, serrilha de com 5 dentes no quarto segmento da cauda e presença de espinho

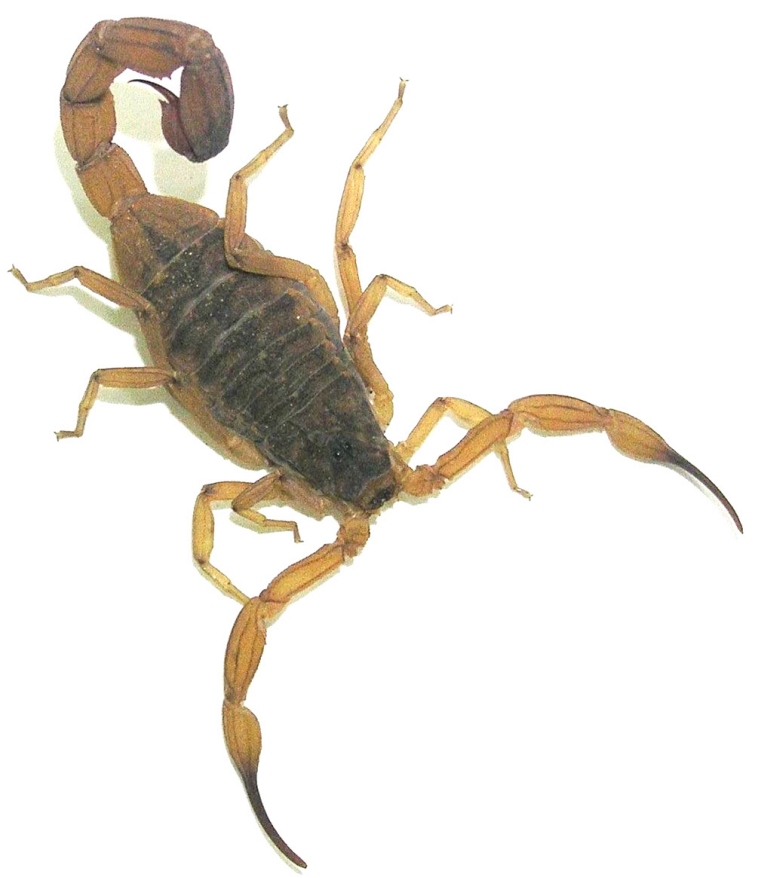

Figura 1. Exemplar de Tityus serrulatus capturado na cidade de Uruguaiana fronteira Oeste do Rio Grande do Sul, Brasil (7,2 cm de comprimento).

Figure 1. Specimen of Tityus serrulatus captured in the city of Uruguaiana West border of Rio Grande do Sul, Brazil (length 7,2 cm).

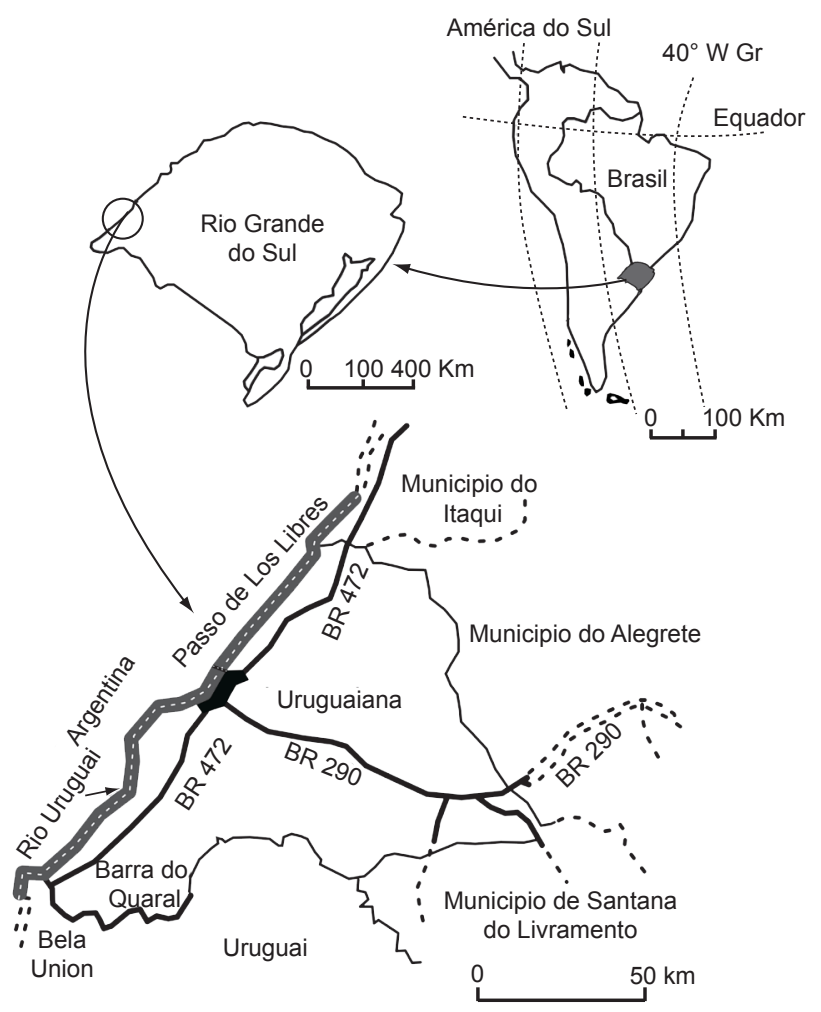

Figura 2. Mapa de localização do município de Uruguaiana, no estado do Rio Grande do Sul, Brasil.

Figure 2. Map of localization of the city of Uruguaiana, in the state of the Rio Grande do Sul, Brazil. 
subaculear no telson. Conforme Lourenço (2002) as medidas são características de indivíduos adultos.

De acordo com Lourenço \& Cuellar (1995); Soerensen (1996), T. serrulatus está distribuído para as regiões nordeste, centro-oeste e sudeste do Brasil. O registro de T. serrulatus para Uruguaiana, região da campanha (RS), corrobora com dados da Funasa (2001), que reporta o grande aumento da dispersão da espécie em relação aos registros anteriores no Brasil.

Conforme Lourenço \& Cloudsley-Thompson, (1999) a espécie é típica de ambientes de mata de transição, florestas secas, cerrados e caatinga. Atualmente, tem sido encontrada em diversos tipos de ambientes incluindo áreas urbanizadas (Soares et al. 2002); T. serrulatus quando encontra condições adequadas possui grande adaptação devido ao fato de apresentar características oportunísticas e ser a única espécie do gênero que se reproduz por partenogênese (Matthiensen 1971, Candido 1999).

A introdução da espécie nos estados de Rondônia e Paraná é relatada por Lourenço \& Eickstead (2003).

Para o Rio Grande do Sul, Torrez et al. (2002), relata um acidente em Porto Alegre ocorrido em um supermercado pela manipulação de produtos hortifrutigranjeiros provenientes de outros estados do Brasil.

O registro da espécie para o oeste do Rio Grande do Sul pode ter ocorrido devido à ação da própria transportadora, uma vez que a mesma atua em vários estados do país. Neste contexto, a dispersão de espécies sinantrópicas vem sendo facilitada pelas atividades antrópicas com o aumento da malha viária do país e o grande fluxo por esta via, o que contribui com ampliação da distribuição original da espécie.

\section{Agradecimentos}

Agradecemos á Dra. Twiggy Cristina Alves Batista da Secretaria de Estado da Saúde do Tocantins, Vigilância Epidemiológica - Programa Contra Acidentes com Animais Peçonhentos, pelo apoio prestado a fins de identificação taxonômica. E ao Laboratório de Biologia da Pucrs Uruguaiana pela disponibilidade do equipamento para registro fotográfico.

\section{Referências Bibliográficas}

BRASIL. 1973. Ministério da Agricultura. Departamento Nacional de Pesquisa Agropecuária. Divisão Pedológica. Levantamento de Reconhecimento dos Solos do Estado do Rio Grande do Sul. Recife, Boletim Técnico $\mathrm{n}^{\circ} .30 .431 \mathrm{p}$.

BRASIL.1986. Ibge. Folha SH.22 Porto Alegre e parte das folhas SH.21 Uruguaiana e SI.22 Lagoa Mirim: geologia, geomorfologia, pedologia, vegetação, uso potencial da terra. Levantamento de Recursos Naturais, 33 Rio de Janeiro, 791p.

BUCARETCHI, F., ZAMBRONE, F.A.D., FONSECA, M.R.C.C., DOUGLAS, J.L. \& TOURINHO, F.S. 1994. Severe scorpion envenomation in children caused by Tityus bahiensis and Tityus serrulatus. In Tel Aviv: International Society on Toxinology (ed). World Congress on Animal, Plant and Microbia Toxins, 11 - Abstracts.

CANDIDO, D. M. 1999. Escorpiões. In Joly CA, Bicudo CEM (eds) Biodiversidade do Estado de São Paulo, Fundação de Amparo à Pesquisa do Estado de São Paulo, São Paulo, p. 25-34. Disponível: http://www.biota. org.br/pdf/v5cap03.pdf (Ultimo acesso em 04/01/07).

CUPO, P., AZEVEDO-MARQUES, M. M., MENEZES, J. B. \& HERING, S. E. 1994a. Escorpionismo. In: BARRAVIERA, B. editor. Venenos animais: uma visão integrada. Rio de Janeiro: EPUC, p.229-312.

CUPO, P., JURCA, M., AZEVEDO-MARQUES, M.M., OLIVEIRA, J.S.M. \& HERING, S.E. 1994b. Severe scorpion envenomation In Brazil.
Clinical, laboratory and anatomopathological aspects. Rev. Inst. Med. Trop. 36:67-76.

CRUZ, E. F. S. 1994a. Biologia dos Escorpiões. In Barraviera, B. Venenos Animais - Uma Visão Integrada. Rio de Janeiro: Editora de Publicações Científicas Ltda. p. 135-150.

EICKSTEDT, V.R.D., CANDIDO, D.M., JORGE, M.T., ALBUQUERQUE, M.J. \& RIBEIRO, L. A. 1994. Escorpionismo no Estado de São Paulo: ocorrência de Tityus serrulatus e T. bahiensis, frequência de acidentes e gravidade dos envenenamentos. Rev. Inst. Med. Trop., 27:56.

FET, V., SISSOM, W.D., LOWE, G. \& BRAUNWALDER., M. E. 1998. Catalogue of scorpions of the world (1758-1997). p. 16, In M. van der Merwe, N.I Platnick \& P. Sierwald (eds.). XIV International Congress of Arachnology - Abstracts.

FET, V., SISSOM, W.D., LOWE, G. \& BRAUNWALDER, M. E. 2000. Catalog of the Scorpions of the World (1758-1998). New York Entomological Society, New York, 690p.

FREIRE MAIA, L., CAMPOS, J.A., AMARAL, C.F. 1994. Approaches to the treatment of scorpion envenoming. Toxicon. v. 32, n. 9, p. 1009-1014.

FUNASA. 2001. Manual de diagnóstico e tratamento de acidentes por animais peçonhentos. Fundação Nacional de Saúde $2^{\mathrm{a}}$ ed. - Brasília. 120p. http://www.ivb.rj.gov.br/palestras/manu_peconhentos.pdf (Ultimo acesso em 04/01/07

FUNDACENTRO. 2001. Fundação Jorge Duprat Figueredo de Segurança e Medicina do Trabalho. Prevenção de Acidente com Animais Peçonhentos. Ministerio do Trabalho e emprego - São Paulo. 49p. http://www.butantan. gov.br/pop/prevencao_acidentes.pdf (Ultimo acesso em 03/01/07).

LOURENÇO, W. R. \& V. R. D. EICKSTEDT. 1988. Considerações sobre a sistemática de Tityus costatus (Karsch, 1879), provável espécie polimórfica de escorpião da floresta atlântica do Brasil (Scorpiones, Buthidae). Iheringia, sér. Zool.,68:3-11.

LOURENÇO, W. R. \& CUELLAR, O. 1995. Scorpions, Scorpionism, Life History Strategies And Parthenogenesis. J. Venom. Anim. Toxins v. 1 n.2. Botucatu.

LOURENÇO, W. R. \& CLOUDSLEY-THOMPSON, J. L. 1999. The Journal of Arachnology. Discovery of a sexual population of Tityus serrulatus, one of the morphs within the complex Tityus stigmurus (Scorpiones; Buthidae). v. 27, p. 154-158.

LOURENÇO, W. R., 2002. Scorpions of Brazil. Les Éditions 1' If. Paris, France. 308p.

LOURENÇO, W.R. \& EICKSTEAD, V.R.D.V. 2003. Escorpiões de Importância Médica. In CARDOSO, J.L.C., FRANÇA, F.O.S., WEN, F.H., MÁLAQUE, C.M.S. \& HADDAD, V. Animais Peçonhentos no Brasil: Biologia, Clínica e Terapêutica dos Acidentes. p. 182-197.

MATTHIENSEN F. A. 1971. The breeding of Tityus. serrulatus Lutz \& Mello, 1922 in captivity (Scorpiones, Buthidae). Rev. Bras. Pesq. Méd. e Biológicas 4:299-300.

SECRETARIA DE SAÚDE DO PARANÁ - Publicações/animais peçonhentos/ Do Centro de Saúde Ambiental. http://saude.pr.gov.br/ftp/Saudeambiental/JVAToxinsV1n21995.rtf (Ultimo acesso em 10/01/07).

SOARES, M. R. M., AZEVEDO, C. S \& MARIA, M. 2002. Escorpionismo em Belo Horizonte, MG: um estudo retrospectivo. Rev. da Soc. Bras. de Méd.Trop. Uberaba MG - Brasil, 35(4):359-363.

SOERENSEN, B. 1996. Animais Peçonhentos - Um Estudo Abrangente: Reconhecimento, Distribuição Geográfica, Produção de Soros, Clínica e Tratamento dos Envenenamentos. Rio de Janeiro: Ed. Atheneu. 138p.

SOLEGLAD, M. E. \& FET, V. 2003. High level systematics and phylogeny of the extant scorpions (Scorpiones: Orthosterni). Escorpius, 11:1-175.

TORRES, J. B., MARQUES, M. G. B. \& MARTINI R, K, E BORGES, C. V. A. 2002. Acidente por Tityus serrulatus e suas implicações epidemiológicas no Rio Grande do Sul. Rev. Saúde Pública; 36(5):631-3. 
